\title{
Effect of Flexi-bar Exercise on Postural Alignment and Balance in Asymmetric Posture
}

\begin{abstract}
This study was conducted to identify how a flexi-bar exercise influences body alignment and balance in adults who have asymmetry in their right or left body. In total, 20 participants were separated into the experimental group and the comparison group. Those in the experimental group participated in a flexi-bar exercise for 6 weeks and based on the coronal plane before and after exercise, their body alignment and balance were measured behind the body. The result was those who had participated in a flexi-bar exercise significantly improved their angle of acromion on both sides, the difference in the angle and height of the posterior superior iliac spine on both sides(p<.05), and the balance of the center sagittal plane(p<.05). Through this study, it could be said that participating in a flexi-bar exercise would improve postural alignment and balance of the shoulder and pelvis in adults with asymmetric posture.
\end{abstract}

Key words: Asymmetric posture; Balance; Flexi-bar; Postural alignment

\author{
Ki Mai Umª, Hyun Sook Kima, In \\ Hyuk Lim

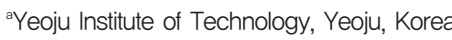 \\ Received : 03 November 2014 \\ Revised : 15 January 2015 \\ Accepted : 17 February 2015 \\ Address for correspondence \\ In Hyuk Lim, PT, Ph.D \\ Department of Physical Therpay, Yeoju \\ Institute of Technology, 338 Sejong-ro, \\ Yeoju, Korea \\ Tel : 82-31-880-5392 \\ E-mail: liminh@yit.ac.kr
}

\section{INTRODUCTION}

The vertebral column provide correct posture and control body movement or all body action to help them work normally(1). However, an abnormal vertebral deformity may be induced due to incorrect posture or long-time computer use during the adolescent growth period, heights of chairs, heavy school bag, and a lack of exercise $(2,3)$. Vertebral deformity related to asymmetric posture incurs outer appearance and functional defect, pain, emotional disorder, and cardiopulmonary disorder and in severe cases, it can cause paralysis and death(4). If an incorrect posture is maintained for a long period, musculoskeletal problem can be introduced(5) and an abnormal posture due to a severe imbalance of muscle strength for a long period could cause abnormal muscle tones in the surrounding muscles, thereby leading to reduced flexibility and pain(6).

Shoulders are the joints that require the most stability in a body with respect to asymmetric posture. Asymmetric shoulder posture due to incorrect habits and posture can increase instability and cause tissue deformity, thereby leading to an increase in loose joint and pathological condition related to stress in other tissues(7). A pelvis is an important region kinematically for movement in a body. It has an anatomical feature that is appropriate for stability during standing, walking, and running and it influences the spine greatly(8). If there are pathological or traumatic problem in the pelvis, such movement will be limited significantly in terms of functional roles(7). A pelvic tilt in the coronal plane of the right or left side can generate asymmetric pressure applied to both gluteal regions, thereby incurring scoliosis(9).

Balance is a process of maintaining postural stability constantly and it is an essential part of human activities to achieve purpose-driven goals(10). Balance is a constant process of maintaining the center of gravity(COG) of the human body on base of support and balance can be achieved by the compensation mechanism in other 
planes while maintaining balance and interaction with the joints(11, 12). As such, imbalance and asymmetric posture can be a cause a functional impairment and balance impairment in the human body, which is why prevention and management are so important.

In recent years, a number of studies on various treatments and exercises related to improvements in asymmetric posture have been conducted(13, 14, 15), but exercise programs that can prevent asymmetric posture should be developed. On the other hand, as a full body exercise method, a flexi-bar exercise has been developed to increase balance and strength in the trunk muscles and body balance in recent years $(16,17)$. A flexi-bar exercise is an exercise method that stimulates multiple muscles in a body by creating $4.6-\mathrm{Hz}$ oscillation, which is appropriate for joint proprioceptive training through active movements using a stick. This exercise improves motion perception not only in healthy persons but also in neurologically impaired patients and it has been reported to have effects on muscle development, involuntary muscle activation, and activation of metabolism thanks to small oscillation and balance control $(16,17)$.

Few studies have been conducted on exercise using a flexi-bar. Thus, this study aimed to study the effects of a flexi-bar exercise on postural alignment and balance with adult subjects in their 20s who had asymmetric posture, thereby developing an effective exercise program for asymmetric posture improvement.

\section{METHODS}

\section{Subjects}

This study was conducted from April 9 to June 10, 2014 for six weeks with students at Y University in Gyeonggi-do. Those who had asymmetric posture behind the body based on the frontal plane were selected as subjects using a Global Postural System(GPS) before the experiment, and those with no other musculoskeletal problems, neurological disorders, or psychiatric problems were selected as 20 final subjects who consented to participate in this study. The general characteristics of subjects are summarized in Table 1.

\section{Measurement}

In this study, the postural alignment and balance of 20 subjects were measured using a GPS to diagnose their postural alignment prior to participating in a flexi-bar exercise program. All subjects were randomly divided into an exercise group of 10 subjects and a control group of 10 subjects. The exercise group performed a flexi-bar (Togu/Germany) exercise for $40 \mathrm{~min}$ daily three times a week for six weeks while the control group maintained daily activities without exercise. Changes in postural alignment and balance improvements in all subjects were measured before and after the exercise.

\section{Body alignment}

A GPS is a full body posture analysis device(18) that can visualize imbalanced postures. In this study, postural alignment in the posterior plane was analyzed. Prior to the GPS measurement, study subjects took off as many items of clothing as needed and a landmark was attached to a specific position(acromion, scapular inferior angle, posterior superior iliac spine[PSIS], and head of ulna) on the subjects. Then, they stood on the assessment bar while looking forward with a relaxed posture. The researcher moved an ideal plumb to position it on the midline of the subject to measure the data. The evaluation of the postural alignment was conducted twice: before and after the study and all data were analyzed using the GPS program.

\section{Balance}

The balance of the subjects was analyzed using 30135/PPpedana stabilometrica(Chinesports, Italy) and a software program called Podate Stabilometric. This equipment can assess balance ability by measuring the COG movements. Subjects

Table 1. General characteristics of subjects

\begin{tabular}{ccccc}
\hline & $\mathrm{n}$ & Age & Height(cm) & Weight(kg) \\
\hline Exercise group & 10 & $20.93 \pm 1.44$ & $170.93 \pm 6.69$ & $65.20 \pm 9.51$ \\
Control group & 10 & $21.07 \pm 2.34$ & $166.87 \pm 9.48$ & $58.87 \pm 9.62$ \\
\hline
\end{tabular}


stood on the area marked in the examination plate with two feet while staring forward. The examination method was as follows: subjects maintained open eyes during measuring of the coronal midpoint, midsagittal midpoint, and curve length of the COG for $1 \mathrm{~min}$.

\section{Exercise program}

The flexi-bar(Togu/Germany) in this study was developed in Germany in the late 1990s. It generates 270 oscillations per $\min (4.6 \mathrm{~Hz})$ and it has been known to be effective for shoulder joints and full body exercises(16). It is divided into a kids bar(green), a standard bar(red), an intensive bar(blue), and an athletic bar(black) depending on the bar strength and vibration force(Flexi-Sports $\mathrm{GmbH}, 200$ ). In this study, a standard bar was used. In this exercise method, the exercise program on the flexi-bar web site was newly configured so the subjects in the exercise group performed each exercise for 30s followed by a 30s rest, which comprised one set. Then, three sets were completed by each subject. In the control group, normal daily activities were done without exercise. The summary of the flexi-bar exercise method is found in Table 2 .

\section{Data analysis}

The collected data produced descriptive statistics about the general postural alignment of subjects using the SPSS 21.0 program for Windows. To compare changes in postural alignment and bal- ance before and after the exercise, a paired t-test was conducted, while an independent t-test was used to compare the difference between the study groups. All statistical significances were analyzed at a level of $\alpha=.05$.

Table 2 Flex-bar program

\begin{tabular}{ccc}
\hline $\begin{array}{c}\text { Warm-up } \\
\text { 10min }\end{array}$ & $\begin{array}{c}\text { Flexi-bar program } \\
\text { exercise 30sec, rest 30sec, 3set }\end{array}$ & $\begin{array}{c}\text { Cool Down } \\
\text { 10min }\end{array}$ \\
\hline & Butterfly front & \\
Spine alignment & \\
Plank front(both) & \\
Stretching & Bridge & Stretching \\
& Deep squat & \\
& Leg stance(both) & \\
& Hunter & \\
& Pelvic floor bent over & \\
\hline
\end{tabular}

\section{RESULTS}

\section{Changes of postural alignment}

To determine the effect of the flexi-bar exercise on postural improvements in adults with asymmetric posture, an analysis based on the sagittal plane was conducted. The result showed a difference in the angle of acromion on both sides and differences in the angle and height of the PSIS on both sides in the exercise group(p<.05). However, the control group had no significant changes statistically(Table 3).

Table 3. Changes of postural alignment

$(\mathrm{M} \pm \mathrm{SD})$

\begin{tabular}{|c|c|c|c|c|c|c|c|c|}
\hline & Group & Pre & Post & $t$ & $p$ & Change & $t$ & $p$ \\
\hline \multirow{2}{*}{$\mathrm{AMH}(\mathrm{cm})$} & $E G$ & $1.32 \pm 0.50$ & $.90 \pm .60$ & 2.522 & .24 & $-.42 \pm .64$ & \multirow{2}{*}{1.295} & \multirow{2}{*}{.206} \\
\hline & $C G$ & $.91 \pm .67$ & $.81 \pm .56$ & .548 & .593 & $-.10 \pm .71$ & & \\
\hline \multirow{2}{*}{$\operatorname{AMA}\left({ }^{\circ}\right)$} & $E G$ & $2.34 \pm .80$ & $1.58 \pm 1.18$ & 2.457 & $.028^{*}$ & $-.76 \pm 1.20$ & \multirow{2}{*}{.764} & \multirow{2}{*}{.451} \\
\hline & $C G$ & $1.83 \pm 1.19$ & $1.41 \pm 1.02$ & 1.246 & .233 & $-.41 \pm 1.29$ & & \\
\hline \multirow{2}{*}{$\mathrm{IFH}(\mathrm{cm})$} & $E G$ & $.86 \pm .39$ & $.90 \pm .66$ & -.255 & .802 & $.04 \pm .61$ & \multirow{2}{*}{-1.251} & \multirow{2}{*}{.221} \\
\hline & $C G$ & $.69 \pm .44$ & $.45 \pm .35$ & 1.503 & .155 & $-.24 \pm .62$ & & \\
\hline \multirow{2}{*}{$\operatorname{IFA}\left({ }^{\circ}\right)$} & $E G$ & $3.53 \pm 2.15$ & $3.43 \pm 2.74$ & .151 & .882 & $-.11 \pm 2.74$ & \multirow{2}{*}{-.574} & \multirow{2}{*}{.570} \\
\hline & $C G$ & $2.50 \pm 1.35$ & $1.89 \pm 1.53$ & 1.195 & .252 & $-.60 \pm 2.00$ & & \\
\hline \multirow{2}{*}{ PSISH(cm) } & $E G$ & $.57 \pm .52$ & $.22 \pm .11$ & 2.542 & $.023^{*}$ & $-.35 \pm .54$ & \multirow{2}{*}{1.274} & \multirow{2}{*}{.213} \\
\hline & $C G$ & $.31 \pm .24$ & $.15 \pm .08$ & 2.102 & .054 & $-.15 \pm .28$ & & \\
\hline \multirow{2}{*}{ PSISA( $\left.{ }^{\circ}\right)$} & $E G$ & $3.97 \pm 1.74$ & $2.35 \pm 1.12$ & 2.764 & $.015^{*}$ & $-1.62 \pm 2.27$ & \multirow{2}{*}{1.574} & \multirow{2}{*}{.127} \\
\hline & $C G$ & $2.16 \pm 1.58$ & $1.86 \pm 1.14$ & .500 & .625 & $-.30 \pm 2.32$ & & \\
\hline
\end{tabular}

${ }^{*} p<.05$

EG : Exercise group, CG : Control group, AMH : Acromion Height, AMA : Acromion Angle, IFH : Inferior Hieht, IFA : Inferior Angle, PSISH :

Posterior Superior Illiac Spine Height, PSISA : Posterior Superior Illiac Spine Angle 


\section{Changes of balance}

To determine the effect of the flexi-bar exercise on postural improvements in adults with asymmetric posture, body balance ability was analyzed as well. The result showed that while opening their eyes, the COG of the exercise group was improved from $-12.48 \pm 4.79$ to $-1.34 \pm 1.87$ in the sagittal plane, indicating a significant movement of the COG to the midpoint(p<.05). However, in the control group, there were no significant changes in the postural improvements and no significant changes were found between the exercise and control groups(Table 4).

Table 3. Changes of balance

$(\mathrm{M} \pm \mathrm{SD})$

\begin{tabular}{|c|c|c|c|c|c|c|c|c|}
\hline & Group & Pre & Post & $t$ & $\mathrm{p}$ & Change & $t$ & $p$ \\
\hline \multirow{2}{*}{$\mathrm{EOCM}(\mathrm{mm})$} & $E G$ & $-1.47 \pm 2.21$ & $-7.77 \pm 3.66$ & 1.550 & .144 & $-6.30 \pm 15.74$ & \multirow{2}{*}{-.109} & \multirow{2}{*}{.914} \\
\hline & $C G$ & $-1.32 \pm 1.61$ & $-9.13 \pm 5.36$ & 1.460 & .168 & $-7.80 \pm 2.00$ & & \\
\hline \multirow{2}{*}{ EOMM(mm) } & $E G$ & $-12.48 \pm 4.79$ & $-1.34 \pm 1.87$ & -2.438 & $.029 *$ & $10.72 \pm 18.48$ & \multirow{2}{*}{1.088} & \multirow{2}{*}{.287} \\
\hline & $C G$ & $-9.69 \pm 5.00$ & $-4.07 \pm 2.91$ & -1.009 & .331 & $5.61 \pm 20.82$ & & \\
\hline \multirow{2}{*}{$\mathrm{EOCL}(\mathrm{mm})$} & $E G$ & $821.667 \pm 60.74$ & $801.20 \pm 63.33$ & .473 & .643 & $-20.46 \pm 167.42$ & \multirow{2}{*}{-1.261} & \multirow{2}{*}{.219} \\
\hline & CG & $676.07 \pm 34.55$ & $733.57 \pm 63.40$ & -1.492 & .160 & $57.50 \pm 144.21$ & & \\
\hline
\end{tabular}

${ }^{*} \mathrm{p}<.05$

EG : Exercise group, CG : Control group, EOCM : Eye Open Coronal Midpoint, EOMM : Eye Open Midsegittal Midpoint, EOCL : Eye Open Curve Length

\section{DISCUSSION}

Asymmetric posture causes trunk deformity due to incorrect postural habits and a lack of exercise, resulting in changes to the body shape(19). In an asymmetric posture, alignment of the vertebral column, shoulder, and pelvis is a very important factor. Asymmetric posture is caused by imbalances of the vertebral column, shoulder, pelvis, and musculoskeletal surrounding. It is known to be prevalent in students or workers who have long sitting hours, indicating a postural abnormality and a collapse in body balance due to maintaining an incorrect posture for a long time $(2,3)$. As a method of improving such a postural abnormality and body imbalance, a flexi-bar exercise has been applied to study subjects. This exercise has been reported to improve the motion perception of not only healthy adults but also neurologically impaired patients. The exercise also affects metabolism activation and balance control due to muscle development, activation of the muscular system, and small oscillation(16, 17). Using the above advantages of the flexi-bar, this study aimed to determine the effects on postural alignment and balance improvements of asymmetric posture.

After the flexi-bar exercise was conducted, differences in the acromion angles on both sides and differences in the height and angle of PSIS on both sides showed improvements. In addition, differences in the angle and height of the scapular inferior angle on both sides and differences in the angle and height of the head of the ulna on both sides showed reduced results compared to the data prior to the experiment. This result verified the flexi-bar exercise could be an effective method to improve asymmetric posture in the pelvis and shoulders. Due to a lack of previous study results on a flexi-bar exercise, this study cannot be compared to other study results directly. However, previous studies on postural improvements with respect to scoliosis patients reported that lumbar stabilization exercises improved asymmetric trunk posture, thereby helping patients to improve their static balance ability(20). Furthermore, yoga exercise was effective in delivering postural improvements to the shoulders and pelvis. The above two results showed that lumbar stabilization exercises and yoga were effective in delivering posture or balance improvements positively.

Through previous studies, we thought that a flexi-bar exercise was effective in improving posture or balance compared to other exercises. Thus, in the future, a comparative study on other exercise methods will be needed.

In a study by Kim, corrective spinal exercises were conducted by female college students with idiopathic scoliosis and the result showed a significant reduction in the Cobb's angle(21). As well, in 
a study by Cho, postural education was given to teenage girls with scoliosis and a spinal correction exercise was conducted. The result showed a reduction in the Cobb's angle and significant flexibility improvements, while the height of PSIS on both sides was the same before $( \pm 4.43)$ and after the exercise $( \pm 4.71)$ after 12 weeks without significant changes(22). In the present study, the height and angle of PSIS on both sides in the flexi-bar exercise group were improved significantly, showing better effects than previous studies. Thus, it is worth studying postural improvements in scoliosis patients in the future.

In the present study, more postural improvements were found than in previous studies when a flexi-bar exercise group conducted the exercise while opening their eyes in terms of balance measurement, where the COG in the midsagittal plane was moved to the midpoint(p<.05). Park conducted a study on a sling exercise and flexibar exercise in chronic lower back pain patients whose ages were between 30 and 60 years and reported significant improvements to right and left balance ability and front and back balance ability(23), which was consistent with the present study result. Thus, Park's result also supported that the flexi-bar exercise was an exercise program effectively delivering postural improvements.

Muscles in the abdomen, waist, and pelvic regions are tonic muscles or postural muscles, which play an important role in lumbar stability and postural control while performing full body exercising $(24,25)$. As the flexi-bar exercise is effective in increasing trunk muscle activity, at the same time(16), such muscle activation plays a role in increasing balance ability effectively.

The present study result cannot be generalized to all adults due to the small number of 20 subjects in a single university and because only adults with asymmetric posture participated in this study. Thus, the study result cannot be generalized to those who were clinically diagnosed with related asymmetric posture. This study also has a limitation in that it did not control postures and habits in individual daily activities. However, this study found that the flexi-bar exercise improved postural alignment and balance ability in adults with asymmetric posture as an exercise program. Thus, we recommend utilizing the flexi-bar exercise program in this study as an exercise method to improve postural alignment.

\section{CONCLUSION}

This study aimed to determine the effect of a flexi-bar exercise on postural alignment and balance improvements, and the following results were observed. The flexi-bar exercise was found to improve the difference in the acromion angle on both sides, the difference in the height and angle of PSIS on both sides, and the balance ability in the sagittal plane. This study verified that the flexi-bar exercise improved the postural alignment and balance ability of those with asymmetric posture.

\section{REFERENCES}

1. Lee SG. Body mass index and balance ability in adolescents with Idiopathic scoliosis. Korea National Sport University 2011.

2. Kang SY. The effects of exercise program on change in curve in girls with mild scoliosis. . Korea University 2002.

3. Lee TH. The effects of Qingong gymnastic exercises and munual manipulation therapy in the correction of bad posture and on improvement of athletic ability of high school girls with scoliosis. Keimyung university 2005.

4. Jo JH, Choi JS, Cho HO, Lee YG, Suck SI. The 'Prevalence of Scoliosis in Junior and Senior High School Students, Pusan, Korea. The Journal of the Korean orthopaedic association 1984; 19(2): 431-435.

5. Park SJ. The Analysis the Changes of Foot Pressure by Various Carrying a Pack Methods during Walking . Daegu University 2009.

6. Kang SH, Lee SH, Lee YS, LeeJC, Jang C, Song MO. The Effect of Balance Training on Shoulder Gradient. Journal of Korean Society of Integrative Medicine 2014; 2(1): 91-100.

7. Neumann, D. Kinesiology of the musculoskeletal system: Foundations for rehabilitation. st louis, MO: Mosby 2010.

8. Panjabi M1, Yamamoto I, Oxland T, Crisco J. How does posture affect coupling in the lumbar spine? Spine 1989; 14(9): 1002-1011.

9. Drummond D, Breed AL, Nerechina R. Relationship of spine deformity and pelvic obliquity on sitting pressure distributions and decubitus ulceration. J Pediartr Orthop 1985; 5(4): 396402. 
10. Wade MG, Jones G. The role of vision and spatial orientation in the maintenance of posture. Phys Ther 1997; 77(6): 619-628.

11. Yaggie JA, McGregor SJ. Effects of isokinetic ankle fatigue on the maintenance of balance and postural limits. Arch Phys Med Rehabil 2002; 83(2): 224-228.

12. Eslami M, Tanaka C, Hinse S, Sebastien K, Farahpour N, Allard P. Effect of foot wedge positions on lower-limb joints, pelvis and trunk angle variability during single-limb stance. The Foot 2006; 16(4): 208-213.

13. Sperandio EF, Alexandre AS, Yi LC, Poletto PR, Gotfryd AO, Vidotto MC, Dourado VZ. Functional aerobic exercise capacity limitation in adolescent idiopathic scoliosis. Spine J 2014; 14(10): 23662372.

14. Choi J, Kim HS, Kim GS Lee HJ, Jeon HS, Chung KM. Posture Management Program Based on Theory of Planned Behavior for Adolescents with Mild Idiopathic Scoliosis. Asian Nurs Res 2013; 7(3): 120-127.

15. Weiss HR. Intervention studies on scoliosis: Review of the reviews. Polish Annals of Medicine. 2012; 19(1): 72-83.

16. Jung JS. Effect of flexi-bar exercise on trunk muscles activity according to posture change in healthy adult. Catholic University of Daegu 2013.
17. Flexi-Sports GmbH. flexibar basics-manual. www.flexi-sports.com. 2008.

18. Yoon SH. The effect of Pilates program for the development of posture and balance. Myongji University 2008.

19. Kwon JH. A study about the dffect of a remedial exercise program for the improvement of cobb's angle in idiopathic scoliosis. Myongji University 2013.

20. Lee WJ. Effect of lumbar stabilization exercise on trunk posture and balance ability in patients with scoliosis. Yonsei University 2011.

21. Kim JE. The effect of corrective spinal exercises on cobb's angle, posture, and core muscle strength according to surface type for female college students. Ewha Womans Universoty 2013.

22. Cho SJ. Effect of spinal correction exercise on teenage girls with scoliosis. Keimyung university 2012.

23. Park HJ. How sling-exercise affects flexibility, balancing ability, body type and pain with patients with chronic lumbar pain. Kookmin University 2013.

24. Akuthota V, Nadler SF. Core strengthening. Arch Phys Med Rehabil 2004; 85(1): 86-92.

25. Marshall PW, Murphy BA. Core stability exercise on and off a swiss ball. Arch Phys Med Rehabil 2005; 86(2): 242-249. 